\title{
Cycle-cocycle partitions and faithful cycle covers for locally finite graphs
}

\author{
Henning Bruhn Reinhard Diestel Maya Stein
}

\begin{abstract}
By a result of Gallai, every finite graph $G$ has a vertex partition into two parts each inducing an element of its cycle space. This fails for infinite graphs if, as usual, the cycle space is defined as the span of the edge sets of finite cycles in $G$. However we show that, for the adaptation of the cycle space to infinite graphs recently introduced by Diestel and Kühn (which involves infinite cycles as well as finite ones), Gallai's theorem extends to locally finite graphs. Using similar techniques we show that if Seymour's faithful cycle cover conjecture is true for finite graphs then it also holds for locally finite graphs when infinite cyles are allowed in the cover, but not otherwise. We also consider extensions to graphs with infinite degrees.
\end{abstract}

\section{Introduction}

By a result of Gallai (see Lovász [9]), every finite graph has a 'cycle-cocycle' partition of its edge set induced by a bipartition of its vertex set:

Theorem 1.1. Every finite graph $G$ admits a vertex partition into (possibly empty) sets $V_{1}, V_{2}$ such that both $E\left(G\left[V_{1}\right]\right)$ and $E\left(G\left[V_{2}\right]\right)$ are elements of the cycle space of $G$.

As stated above, Gallai's theorem has no obvious extension to infinite graphs. Indeed, when $G$ is infinite, the elements of its (combinatorial) cycle space are still finite sets of edges, so a partition as in Theorem 1.1 does not exist, for instance, when $G$ is an infinite disjoint union of triangles.

One way to deal with the problem is to look for an equivalent reformulation of Theorem 1.1 and extend that. For example:

Theorem 1.2. Every locally finite graph $G$ admits a vertex partition into (possibly empty) sets $V_{1}, V_{2}$ such that in both $G\left[V_{1}\right]$ and $G\left[V_{2}\right]$ all vertex degrees are even.

(The proof of Theorem 1.2 is an easy exercise in compactness. It is also an immediate corollary of Theorem 1.4 below.)

However, the requirement that all degrees of a subgraph $H$ of a finite graph $G$ should be even is only one equivalent reformulation among many of saying that $E(H)$ lies in the cycle space of $G$. Another is that $H$ should be an edgedisjoint union of cycles (and isolated vertices). This would be just as meaningful for infinite $H$, and for locally finite $H$ it implies the even-degree condition but 
not conversely. (Consider a double ray, which is 2-regular but not a union of cycles.) But with this latter reformulation, Theorem 1.1 no longer extends to infinite graphs:

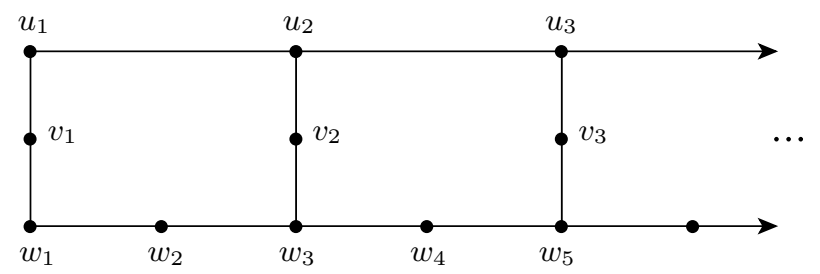

Figure 1: A graph with no bipartition into edge-disjoint unions of cycles

Example 1.3. The graph $G$ shown in Figure 1 has a unique vertex partition into two induced even-degree subgraphs. One of these is edgeless, the other a double ray.

Proof. Consider any partition $\left(V_{1}, V_{2}\right)$ of $V(G)$. Note that if two vertices $x, y$ (such as $u_{1}$ and $w_{1}$ ) have a common neighbour $z$ (such as $v_{1}$ ) not adjacent to any other vertex, then $x$ and $y$ must lie in the same partition class: otherwise, $z$ would have degree 1 in its partition class. Thus if $u_{1} \in V_{1}$, say, we deduce inductively that $w_{1}, w_{3}, w_{5}, \ldots \in V_{1}$ and hence also $u_{2}, u_{3}, u_{4}, \ldots \in V_{1}$. But $u_{2}, u_{3}, u_{4} \ldots$ must not have degree 3 in $G\left[V_{1}\right]$, so $v_{2}, v_{3}, v_{4}, \ldots \in V_{2}$. Finally, $v_{1}$ lies in $V_{1}$ because $u_{2}$ does, so inductively $w_{2}, w_{4}, \ldots \in V_{1}$.

Thus, $V_{2}$ is the independent set $\left\{v_{2}, v_{3}, \ldots\right\}$, while $V_{1}$ consists of the remaining vertices, which span a double ray.

Our aim in this paper is to show that, despite Example 1.3, Theorem 1.2 is not the strongest possible extension of Theorem 1.1. Indeed, we can say more of the double ray $G\left[V_{1}\right]$ in Figure 1 than that its degrees are even: the double ray forms an infinite cycle in the topological cycle space $\mathcal{C}(G)$ introduced for infinite graphs in [3, 7]. (It does so, because its tails converge to the same end of $G$, which thus 'closes it up'; see Section 2 for formal definitions.) So for that space $\mathcal{C}(G)$, the graph of Figure 1 is no longer a counterexample to Theorem 1.1. And indeed, we have the following extension of Theorem 1.1 to infinite graphs, which implies Theorem 1.2 but is quite a bit stronger:

Theorem 1.4. For every locally finite graph $G$ there is a partition of $V(G)$ into two (possibly empty) sets $V_{1}, V_{2}$ such that $E\left(G\left[V_{i}\right]\right) \in \mathcal{C}(G)$ for both $i=1,2$.

We shall prove Theorem 1.4 in Section 3. In Section 4 we use similar techniques to extend the cycle double cover conjecture and Seymour's faithful cycle cover conjecture to locally finite graphs: if these conjectures are true for finite graphs, they also hold for locally finite graphs with our notion of an infinite topological cycle space. (The latter conjecture fails unless infinite cycles are admitted; for the former we have been unable to decide whether infinite cycles are really needed.) In Section 5 we generalize our results to graphs with infinite degrees, as far as this can be reasonably expected. 


\section{Definitions}

In this section we briefly define those of our terms that are not commonly known. A more detailed introduction covering all these, as well as standard terms not defined below, can be found in [5].

When $A$ is a set, we write $\bigcup A$ for the union of all its elements. A 1-way infinite path is called a ray, a 2-way infinite path is a double ray, and the subrays of a ray or double ray are its tails. Let $G=(V, E)$ be any graph. Two rays in $G$ are equivalent if no finite set of vertices separates them; the corresponding equivalence classes of rays are the ends of $G$. We denote the set of these ends by $\Omega=\Omega(G)$.

Let us define a topology on $G$ together with its ends. We shall call this topology VTOP; if $G$ is locally finite, then this topology is usually called its Freudenthal compactification. We begin by viewing $G$ itself (without ends) as the point set of a 1-complex. Then every edge is a copy of the real interval $[0,1]$, and we give it the corresponding metric and topology. For every vertex $v$ we take as a basis of open neighbourhoods the open stars of radius $1 / n$ around $v$. (That is to say, for every integer $n \geq 1$ we declare as open the set of all points on edges at $v$ that have distance less than $1 / n$ from $v$, in the metric of that edge. $)^{1}$ In order to extend this topology to $\Omega$, we take as a basis of open neighbourhoods of a given end $\omega \in \Omega$ the sets of the form

$$
C(S, \omega) \cup \Omega(S, \omega) \cup \stackrel{\circ}{E}(S, \omega),
$$

where $S \subseteq V$ is a finite set of vertices, $C(S, \omega)$ is the unique component of $G-S$ in which every ray in $\omega$ has a tail, $\Omega(S, \omega)$ is the set of all ends $\omega^{\prime} \in \Omega$ whose rays have a tail in $C(S, \omega)$, and $\stackrel{\circ}{E}(S, \omega)$ is the set of all inner points of edges between $S$ and $C(S, \omega) .^{2}$ Let $|G|$ denote the topological space on the point set $V \cup \Omega \cup \cup E$ thus defined. We shall freely view $G$ and its subgraphs either as abstract graphs or as subspaces of $|G|$. Note that in $|G|$ every ray converges to the end of which it is an element.

A set $C \subseteq|G|$ is a circle if it is homeomorphic to the unit circle. Then $C$ includes every edge of which it contains an inner point, and the graph consisting of these edges and their endvertices is the cycle defined by $C$. Conversely, it is not hard to show [7] that $C \cap G$ is dense in $C$, so every circle is the closure in $|G|$ of its cycle and hence defined uniquely by it. Note that every finite cycle in $G$ is also a cycle in this sense, but there can also be infinite cycles; see $[3,5]$ for examples and for more information on VTOP and $\mathcal{C}(G)$.

Call a family $\left(D_{i}\right)_{i \in I}$ of subsets of $E$ thin if no vertex of $G$ is incident with an edge in $D_{i}$ for infinitely many $i$. (Thus in particular, no edge lies in more than finitely many $D_{i}$.) Let the sum $\sum_{i \in I} D_{i}$ of this family be the set of all edges that lie in $D_{i}$ for an odd number of indices $i$, and let the topological cycle space $\mathcal{C}(G)$ of $G$ be the set of all sums of (thin families of) edge sets of cycles, finite or

\footnotetext{
${ }^{1}$ If $G$ is locally finite, this is the usual identification topology of the 1-complex. Vertices of infinite degree, however, have a countable neighbourhood basis in VTOP, which they do not have in the 1-complex.

${ }^{2}$ In the early papers on this topic, such as $[7,8,6]$, some more basic open sets were allowed: in the place of $\stackrel{\circ}{E}(S, \omega)$ we could take an arbitrary union of open half-edges from $C$ towards $S$ one from every $S-C$ edge. When $G$ is locally finite, this yields the same topology. When $G$ has vertices of infinite degree, our topology is slightly sparser but still yields the same topological cycle space; see the end of this section for more discussion.
} 
infinite. Symmetric difference as addition makes $\mathcal{C}(G)$ into an $\mathbb{F}_{2}$ vector space, which coincides with the usual cycle space of $G$ when $G$ is finite. We remark that $\mathcal{C}(G)$ is closed also under taking infinite thin sums $[7,8]$, which is not obvious from the definitions.

As with finite graphs, elements of $\mathcal{C}(G)$ can be decomposed into cycles:

Theorem 2.1. [8] Every element of the topological cycle space of a graph is the edge-disjoint union of cycles.

For the conscientious reader we remark that, although the topology for $|G|$ considered in $[7,8,6]$ is slightly larger than ours (see the earlier footnote), the above theorem, as well as Lemma 3.1 below, is nevertheless applicable in our context. This is because the cycles in $|G|$ coincide for these topologies: as one readily checks, the identity on $|G|$ between the two spaces is bicontinuous when restricted to a circle in either space.

\section{Cycle-cocycle partitions}

The purpose of this section is to prove Theorem 1.4. This proof will also serve as a model for other proofs later in the paper, which will refer to this proof and skip the corresponding details.

Our proof of Theorem 1.4 will be a compactness proof, but we shall need a non-trivial lemma from [7] to make this possible. Recall that while Theorem 1.2 has a straightforward compactness proof, the naïve extension of Theorem 1.1 to locally finite graphs does not (and is in fact false). The reason is, roughly speaking, that having all degrees even is a 'local' property of finite subsets $S \subseteq V(G)$ (one that $S$ will satisfy in every large enough induced subgraph or in none), while inducing part of an element of the (combinatorial) cycle space based on finite cycles is not: the sequence of finite cycles $C_{n}=P_{n}+e_{n}$, for example, where the $P_{n}=v_{-n} v_{-(n-1)} \ldots v_{n-1} v_{n}$ are nested paths and $e_{n}$ is the edge $v_{-n} v_{n}$, 'tends' for $n \rightarrow \infty$ to the double ray $D=\ldots v_{-1} v_{0} v_{1} \ldots$ whose edge set does not lie in the combinatorial cycle space of $\bigcup_{n \in \mathbb{N}} C_{n}$. However, $D$ is an infinite cycle in $\bigcup_{n \in \mathbb{N}} C_{n}$, and more generally it turns out that all such 'limits' of finite cycles in a graph $G$ are elements of $\mathcal{C}(G)$ (though not necessarily single infinite cycles).

The following result from [7] makes that precise by providing a characterization of the elements of $\mathcal{C}(G)$ among all the subsets of $E(G)$ that is 'local' in the above sense.

Lemma 3.1. [7] Let $G$ be a locally finite graph. Then the following statements are equivalent for every $Z \subseteq E(G)$ :

(i) $Z \in \mathcal{C}(G)$; and

(ii) $|F \cap Z|$ is even for every finite cut $F$ of $G$.

We shall cast our compactness proof in terms of König's infinity lemma (see [5]), which we restate:

Lemma 3.2. Let $W_{1}, W_{2}, \ldots$ be an infinite sequence of disjoint non-empty finite sets, and let $H$ be a graph on their union. For every $n \geq 2$ assume that every vertex in $W_{n}$ has a neighbour in $W_{n-1}$. Then $H$ contains a ray $v_{1} v_{2} \ldots$ with $v_{n} \in W_{n}$ for all $n$. 
Proof of Theorem 1.4. By treating the components of $G$ separately, we may assume that $G$ is connected. Hence, being locally finite, $G$ is countable. Let $v_{1}, v_{2}, \ldots$ be an enumeration of $V(G)$. For $n \in \mathbb{N}$ set $S_{n}:=\left\{v_{1}, \ldots, v_{n}\right\}$, and define $W_{n}$ as the set of all tuples $\left(V_{1}, V_{2}\right)$ such that

(i) $\left(V_{1}, V_{2}\right)$ is a partition of $S_{n}$ into two (possibly empty) sets; and

(ii) for $i=1,2$, there is a $Z \in \mathcal{C}(G)$ such that $Z \cap E\left(G\left[S_{n}\right]\right)=E\left(G\left[V_{i}\right]\right)$.

Each set $W_{n}$ is clearly finite. It is non-empty by Theorem 1.1 applied to $G\left[S_{n}\right]$.

Let us define a graph $H$ on $\bigcup_{n=1}^{\infty} W_{n}$. For $n \geq 2$, let $\left(V_{1}, V_{2}\right) \in W_{n}$ be adjacent to $\left(V_{1}^{\prime}, V_{2}^{\prime}\right) \in W_{n-1}$ if and only if, for both $i=1,2, V_{i}^{\prime} \subseteq V_{i}$. Observe that for $n \geq 2$ every vertex in $W_{n}$ has a neighbour in $W_{n-1}$.

By the infinity lemma (3.2), there is a ray $v_{1} v_{2} \ldots$ in $H$ with $\left(V_{1}^{n}, V_{2}^{n}\right):=$ $v_{n} \in W_{n}$ for all $n$. Clearly, $V_{1}:=\bigcup_{n=1}^{\infty} V_{1}^{n}$ and $V_{2}:=\bigcup_{n=1}^{\infty} V_{2}^{n}$ form a partition of $V(G)$.

We shall use Lemma 3.1 to show that $E\left(G\left[V_{1}\right]\right) \in \mathcal{C}(G)$, and in a similar way that $E\left(G\left[V_{2}\right]\right) \in \mathcal{C}(G)$. Write $Z_{n}:=E\left(G\left[V_{1}^{n}\right]\right)$ for each $n$. Consider a finite cut $F$ of $G$. Choose $n$ large enough that $F \subseteq E\left(G\left[S_{n}\right]\right)$. By (ii), there is a $Z \in \mathcal{C}(G)$ with $Z \cap E\left(G\left[S_{n}\right]\right)=Z_{n}$. Then

$F \cap E\left(G\left[V_{1}\right]\right)=F \cap E\left(G\left[S_{n}\right] \cap G\left[V_{1}\right]\right)=F \cap Z_{n}=F \cap Z \cap E\left(G\left[S_{n}\right]\right)=F \cap Z$.

Since $Z \in \mathcal{C}(G)$, the last intersection is even. Hence $E\left(G\left[V_{1}\right]\right) \in \mathcal{C}(G)$ by Lemma 3.1, as desired.

\section{Faithful cycle covers}

Another problem concerning cycles is the well-known cycle double cover conjecture, which states that every bridgeless finite graph has a cycle double cover. (A cycle double cover of a graph $G$ is a family of cycles such that each edge of $G$ lies on exactly two of those cycles.) Using the same techniques as in the proof of Theorem 1.4 one can show that if the cycle double cover conjecture is true for finite graphs then it also holds for locally finite graphs, possibly with infinite cycles. However, we have been unable to construct an example where infinite cycles are really needed.

The situation is different for the following related conjecture of Seymour, which extends with infinite cycles but fails with finite cycles only. For a graph $G$ and a map $p: E(G) \rightarrow \mathbb{N}(\ni 0)$ a faithful cycle cover of $(G, p)$ is a family of cycles such that every edge $e \in G$ lies on exactly $p(e)$ of those cycles. Such a map $p$ is admissible if $p(F)=\sum_{f \in F} p(f)$ is even and $p(e) \leq p(F) / 2$ for every finite cut $F$ and every edge $e \in F$. We call $p$ even if all its values $p(e)$ are even numbers. If $(G, p)$ is to have a faithful cycle cover, then obviously $p$ has to be admissible, and we shall see below that for some $G$ it has to be even. Since the constant map with value 2 is admissible for bridgeless graphs, the following faithful cycle cover conjecture extends the cycle double cover conjecture:

Conjecture 4.1 (Seymour [10]). Let $G$ be a finite graph, and $p$ an even admissible map. Then $(G, p)$ has a faithful cycle cover.

Unlike the cycle double cover conjecture, we know that Conjecture 4.1 fails for locally finite graphs unless we allow infinite cycles. Here is a simple example. 
Let $G$ be the double (= two-way infinite) ladder, and let $p$ assign 0 to every rung and 2 to all the other edges. By our current definition of admissibility (which requires $p(e) \leq p(F) / 2$ only for finite cuts $F$ ), the function $p$ is admissible. But $G$ contains no finite cycle that avoids all rungs, so $(G, p)$ has no faithful cover consisting of finite cycles. (It does, however, have a faithful cover consisting of two copies of the infinite cycle spanned by the edges for which $p=2$.)

The above example is no longer a counterexample to the infinite analogue of Conjecture 4.1 if we require of an admissible map $p$ that it satisfies $p(e) \leq p(F) / 2$ also for infinite cuts $F$ (and edges $e \in F$ ): if $e$ is any edge with $p(e)=2$ and $R$ is a maximal ray in the subgraph of $G-e$ spanned by all its remaining edges with $p=2$, then $e$ and the edges with $p=0$ incident with $R$ form an infinite cut $F$ such that $p(e)=p(F)$. Thus, $p$ is no longer admissible, and we no longer have a contradiction.

Our next example, however, shows that strengthening the definition of 'admissible' as above is not enough to make Conjecture 4.1 true for locally finite graphs - if only finite cycles are admitted. Consider the ladder $G$ shown in Figure 2 and the admissible map $p: E(G) \rightarrow \mathbb{N}$ defined by $p\left(e_{i}\right)=p\left(e_{i}^{\prime}\right)=2 i$ and $p\left(f_{i}\right)=2$ for all $i$. (Since $p(e)>0$ for all $e$, we trivially have $p(e) \leq p(F) / 2$ also for infinite cuts $F$.) Suppose there is a faithful cycle cover which contains a finite cycle $D$. Obviously, $D$ contains exactly two rungs $f_{m}, f_{n}$, with $m<n$, say. Let $\mathcal{C}$ be the subfamily of the cover consisting of those cycles which pass through the edge $e_{n}$. Each but at most one (which might go through $f_{n}$ ) of the cycles in $\mathcal{C}$ must use the edge $e_{n-1}$. Thus, at least $|\mathcal{C}|-1=2 n-1$ cycles of the cover meet the edge $e_{n-1}$, contradicting $p\left(e_{n-1}\right)=2 n-2$. Therefore, the only faithful cycle cover that $(G, p)$ can have (and which is easily seen to exist) must be one consisting of infinite cycles.

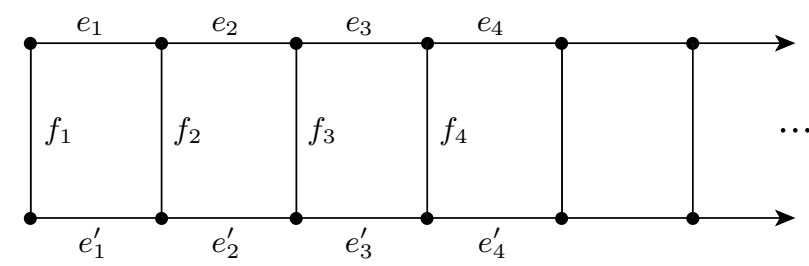

Figure 2: The unique faithful cycle cover consists of infinite cycles only

As soon as we allow infinite cycles, however, Conjecture 4.1 does extend to locally finite graphs:

Theorem 4.2. Let $G$ be a locally finite graph and $p: E(G) \rightarrow \mathbb{N}$ an even admissible map. If Conjecture 4.1 is true then $(G, p)$ has a faithful cycle cover.

Proof. We sketch how the proof of Theorem 1.4 has to be amended for Theorem 4.2. As before, we may assume that $G$ is connected. Let $v_{1}, v_{2}, \ldots$ be an enumeration of its vertices, and set $G_{n}:=G\left[\left\{v_{1}, \ldots, v_{n}\right\}\right]$. We define $W_{n}$ as the set of all families $\mathcal{E}$ of edge sets $E \subseteq E\left(G_{n}\right)$ such that

(i) every edge $e \in G_{n}$ lies in exactly $p(e)$ members of $\mathcal{E}$; and

(ii) for every $E \in \mathcal{E}$ there is a finite cycle $C \subseteq G$ with $E\left(C \cap G_{n}\right)=E$. 
The sets $W_{n}$ are nonempty. Indeed, consider the multigraph obtained by contracting the components of $G-G_{n}$ to one vertex each, keeping parallel edges but deleting loops. Subdividing the parallel edges we obtain a simple finite graph $G_{n}^{\prime}$. The map $p$ induces an even and admissible map on $G_{n}^{\prime}$, for which there is a faithful cycle cover by assumption. It is easy to see that the corresponding edges in $G$ satisfy (i) and (ii).

The rest of the proof is analogous to that of Theorem 1.4: applying the infinity lemma to an auxiliary graph $H$, we obtain a family of elements of $\mathcal{C}(G)$ such that every edge $e$ lies on exactly $p(e)$ members of this family. By Theorem 2.1, we can modify this into a faithful cover consisting of single cycles. Therefore, if the faithful cycle cover conjecture holds for finite graphs, it is also true for locally finite graphs.

Conjecture 4.1 requires $p$ to be even, and indeed if $p$ is allowed to assume odd values the conjecture becomes false: take the Petersen graph, and give $p$ the value 2 on a perfect matching and 1 on all other edges.

Take any subgraph of an infinite graph $G$, and contract some-possibly infinitely many - of its edges; the resulting graph will be called a minor of $G$. Then the following result, whose finite version is a theorem of Alspach, Goddyn and Zhang [1], can be proved like Theorem 4.2.

Theorem 4.3. Let $G$ be a locally finite graph not containing the Petersen graph as a minor, and let $p: E(G) \rightarrow \mathbb{N}$ be any admissible map (even or not). Then $(G, p)$ has a faithful cycle cover.

\section{$5 \quad$ Graphs with infinite degrees}

Theorem 1.4 does not extend to arbitrary graphs with vertices of infinite degree. For example, consider the graph $G$ obtained by joining a vertex $v_{0}$ to every vertex of a ray $R:=v_{1} v_{2} v_{3} \ldots$. Suppose there is a partition as in Theorem 1.4, and assume that $v_{0} \in V_{1}$. By the definition of thin sums, no element of $\mathcal{C}(G)$ can have infinitely many edges incident with $v_{0}$. So there is a maximal $n \geq 0$ with $v_{n} \in V_{1}$. But then $v_{n+1}$ has degree 1 in $G\left[V_{2}\right]$, a contradiction.

The problem here is that no element of the topological cycle space is allowed to have a vertex of infinite degree. Indeed if we weaken our concept of infinite sums, forbidding only those where some edge lies in infinitely many of the summands (i.e. making no restrictions on vertices), our counterexample ceases to be one: for $V_{1}:=\left\{v_{3}, v_{6}, v_{9}, \ldots\right\}$, the set $V_{2}:=V \backslash V_{1}$ induces an element of the cycle space. Of course, there was a good reason for forbidding these sums: summing up the triangles $v_{0} v_{1} v_{2} v_{0}, v_{0} v_{2} v_{3} v_{0}, v_{0} v_{3} v_{4} v_{0}, \ldots$ yields the ray $v_{0} v_{1} R$, which should then also be a member of the cycle space. But this is not unreasonable: as $v_{0}$ cannot be separated finitely from the ray $R$, this ray may be seen as converging to $v_{0}$. Indeed, although $R$ does not converge to $v_{0}$, it nearly does: VTOP cannot separate its end from $v_{0}$ by two disjoint open sets. If we adjust our topology so that $R$ does converge against $v_{0}$, by identifying $v_{0}$ with the end containing $R$, the ray $v_{0} v_{1} R$ becomes a cycle as desired.

Let us make that precise. We say that a vertex $v$ dominates an end $\omega$ in $G$ if there is ray $R \in \omega$ and an infinite set of $v-R$ paths that meet pairwise only in $v$. Assuming that

every end of $G$ is dominated by at most one vertex, 
we now identify each vertex with all the ends it dominates, to obtain space $\tilde{G}$ whose (quotient) topology we denote by ITOP. Note that, by (1), the vertices of $G$ remain distinct in this identification. The identification space $\tilde{G}$ is Hausdorff (unlike $|G|$, when $G$ has a dominated end), and compact [4] if $G$ is 2-connected and satisfies condition (2) below. See $[2,6]$ for more on ITOp. ${ }^{3}$

To obtain a cycle space which retains the natural properties of the topological cycle space of a locally finite graph, we have to impose another restriction on our graph $G$. Indeed, consider two vertices $x$ and $y$ that are linked by infinitely many independent paths. Then we can generate each of these paths $P$ as a sum of cycles, so $P$ should be in our cycle space. To avoid this, we require the following:

No two vertices of $G$ are joined by infinitely many independent paths.

Note that (2) implies (1). As before, we define as cycles those subgraphs of $G$ whose closure in $\tilde{G}$ is homeomorphic to the unit circle, and the topological cycle space $\mathcal{C}(\tilde{G})$ of $\tilde{G}$ is defined as the span of all sums of cycles such that no edge appears in infinitely many of the summands. For the rest of this section, we assume that the graphs $G$ we consider satisfy (2), and that all cycles are defined with respect to $\tilde{G}$.

Our main result now extends to graphs with infinite degrees, as follows:

Theorem 5.1. Let $G$ be a graph satisfying (2). Then there is a partition of $V(G)$ into two (possibly empty) sets $V_{1}, V_{2}$ such that $E\left(G\left[V_{i}\right]\right) \in \mathcal{C}(\tilde{G})$ for both $i=1,2$.

For the proof of Theorem 5.1 we may assume $G$ to be 2 -connected, because the topological cycle space of a graph is the direct product of the topological cycle spaces of its blocks. (Recall that vertices are now allowed to lie in infinitely many summands as long as no edge does.) Then $G$ is countable [6]. We now proceed exactly as in the proof of Theorem 1.4, except that instead of Lemma 3.1 we use the following analogous result:

Lemma 5.2. [6] Let $G$ be a graph satisfying (2). Then $\mathcal{C}(\tilde{G})$ consists of precisely those sets of edges that meet every finite cut in an even number of edges.

Our results of Section 4 can also be extended to graphs with infinite degrees, but we require the following strengthening of (2):

No two vertices of $G$ are joined by infinitely many edge-disjoint paths.

This is indeed stronger than (2), see [6].

We need another lemma.

Lemma 5.3. Let $G$ be a 2-connected multigraph satisfying (3), and let $U$ be a finite set of vertices in $G$. Then we can contract edges of $G$, deleting loops but keeping any multiple edges that arise, so that no two vertices from $U$ are identified, the multigraph $H$ obtained has only finitely many edges and vertices, and every cut of $H$ is also a cut of $G$.

\footnotetext{
${ }^{3}$ Here, we obtain ITOP from VTOP, which is slightly sparser than the topology ToP from which ITOP is derived in [6]. However, in a similar way as detailed at the end of Section 2, we see that both topologies yield the same cycle space. In particular, Lemma 5.2 is still applicable.
} 
Proof. First, note that the set $\mathcal{K}$ of components of $G-U$ is finite. Indeed, as $G$ is 2-connected, every component $C$ of $G-U$ has distinct neighbours $u, v$ in $U$. If $\mathcal{K}$ is infinite, then infinitely many $C \in \mathcal{K}$ are joined to the same two vertices $u, v$ (because $U$ is finite), so these are linked by infinitely many independent paths. This contradicts (3).

Next, consider a component $C \in \mathcal{K}$. For every two vertices $u, v \in U$ that both send infinitely many edges to $C$ there is a finite cut $F_{u, v} \subseteq E(C)$ separating $N(u) \cap V(C)$ from $N(v) \cap V(C)$ in $C$, because of (3). Let $F_{C}$ be the union of all such cuts $F_{u, v}$. Note that $F_{C}$ is finite, as there are only finitely many pairs $u, v$. Then the set $\mathcal{K}_{C}$ of components of $C-F_{C}$ is also finite, and so is $\mathcal{K}^{\prime}:=\bigcup_{C \in \mathcal{K}} \mathcal{K}_{C}$. Each $D \in \mathcal{K}^{\prime}$ sends only finitely many edges to $G-U-D$, and at most one vertex in $U$ sends infinitely many edges to $D$. If such a vertex exists, we denote it by $u_{D}$.

In $G$, contract every $D \in \mathcal{K}^{\prime}$ to a vertex $v_{D}$, keeping parallel edges but deleting loops. If two vertices of the resulting multigraph are joined by infinitely many edges, then these are $u_{D}$ and $v_{D}$ for some $D \in \mathcal{K}^{\prime}$. In a second step, we now contract all these edges $u_{D} v_{D}$, again keeping parallel edges. We obtain a finite multigraph $H$ in which no two vertices from $U$ are identified. (Note in particular that the edge set of $H$ is finite, despite the parallel edges that arose in the contraction.) Since we did not delete any edges except loops, every cut of $H$ is also a cut of $G$.

Theorem 5.4. Let $G$ be a graph satisfying (3), and let $p: E(G) \rightarrow \mathbb{N}$ be an even admissible map. If Conjecture 4.1 is true then $(G, p)$ has a faithful cycle cover.

Proof. Consider a block $B$ of $G$. Every cut of $B$ is a cut of $G$, so the restriction of $p$ to $B$ is an even admissible map on $B$. As $\mathcal{C}(G)$ is the direct product of the topological cycle spaces of the blocks of $G$, we may therefore assume $G$ to be 2-connected. (Note that $p$ assigns zero to bridges, so we need not cover these.) Then $G$ is countable [6].

Consider an enumeration $v_{1}, v_{2}, \ldots$ of $V(G)$, and set $G_{n}:=G\left[\left\{v_{1}, \ldots, v_{n}\right\}\right]$. Define $W_{n}$ as the set of all families $\mathcal{E}$ of sets $E \subseteq E\left(G_{n}\right)$ such that

(i) every edge $e \in E\left(G_{n}\right)$ lies in exactly $p(e)$ members of $\mathcal{E}$; and

(ii) for every $E \in \mathcal{E}$ there is a finite cycle $C \subseteq G$ with $E\left(C \cap G_{n}\right)=E$.

Let us show that the sets $W_{n}$ are not empty. Apply Lemma 5.3 with $U=$ $\left\{v_{1}, \ldots, v_{n}\right\}$, and denote the multigraph $H$ obtained by $G_{n}^{\prime}$. Since every cut of $G_{n}^{\prime}$ is also one of $G$, the map $p$ induces an admissible even map $p_{n}^{\prime}$ on $G_{n}^{\prime}$. By subdividing edges we obtain from $G_{n}^{\prime}$ a simple graph $G_{n}^{\prime \prime}$ with admissible even map $p_{n}^{\prime \prime}$ (induced by $p_{n}^{\prime}$ ). Then by assumption there is a faithful cycle cover of $\left(G_{n}^{\prime \prime}, p_{n}^{\prime \prime}\right)$. Every cycle in that cover can be extended to a finite cycle in $G$. The family of these cycles then satisfies (i) and (ii), thus proving $W_{n} \neq \emptyset$.

The rest of the proof is again analogous to that of Theorem 1.4, since every element of $\mathcal{C}(\tilde{G})$ is an edge-disjoint union of cycles [6].

Using the same techniques as above, we can also extend Theorem 4.3:

Theorem 5.5. Let $G$ be a graph that satisfies (3) and does not contain the Petersen graph as a minor, and let $p: E(G) \rightarrow \mathbb{N}$ be any admissible map. Then $(G, p)$ has a faithful cycle cover. 


\section{References}

[1] B. Alspach, L. Goddyn, and C-Q. Zhang. Graphs with the circuit cover property. Trans. Am. Math. Soc., 344:131-154, 1994.

[2] H. Bruhn and R. Diestel. Duality in infinite graphs. To appear in Comb., Probab. Comput..

http://www.math. uni-hamburg. de/math/research/preprints/hbm.html.

[3] R. Diestel. The cycle space of an infinite graph. To appear in Comb., Probab. Comput.

[4] R. Diestel. End spaces and spanning trees. Submitted. http://www.math.uni-hamburg. de/math/research/preprints/hbm.html.

[5] R. Diestel. Graph Theory (3rd ed., in preparation). Springer-Verlag, 2005.

[6] R. Diestel and D. Kühn. Topological paths, cycles and spanning trees in infinite graphs. To appear in Europ. J. Combinatorics.

[7] R. Diestel and D. Kühn. On infinite cycles I. Combinatorica, 24:69-89, 2004.

[8] R. Diestel and D. Kühn. On infinite cycles II. Combinatorica, 24:91-116, 2004.

[9] L. Lovász. Combinatorial Problems and Exercises (2nd edition). NorthHolland, 1993.

[10] P.D. Seymour. Sums of circuits. In J.A. Bondy and U.S.R. Murty, editors, Graph Theory and Related Topics, pages 341-355. Academic Press, 1979.

Version 22 November 2004

Henning Bruhn <hbruhn@nullsinn. net>

Reinhard Diestel <diestel@math.uni-hamburg.de>

Maya Stein <fm7y052@public.uni-hamburg.de>

Mathematisches Seminar

Universität Hamburg

Bundesstraße 55

20146 Hamburg

Germany 